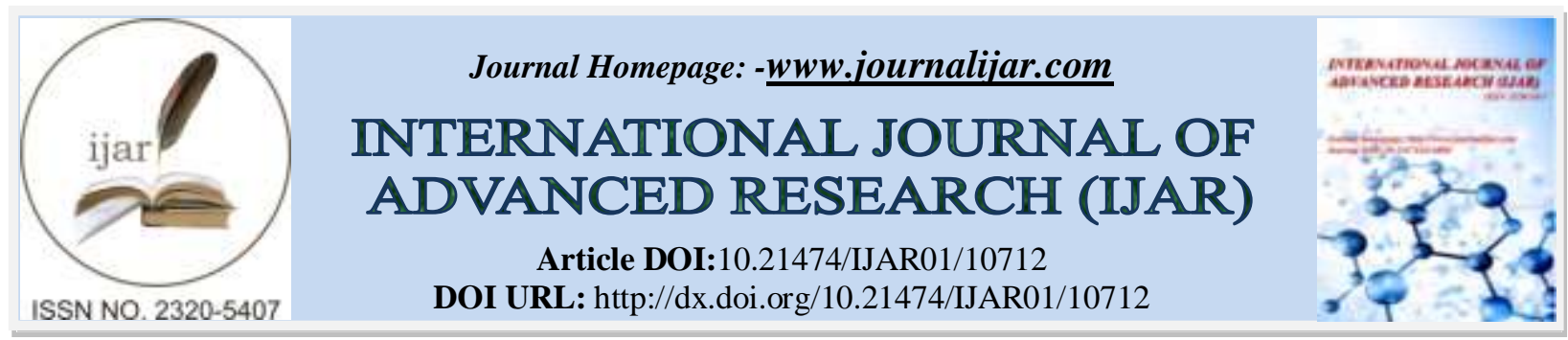

RESEARCH ARTICLE

\title{
STATUS OF VITAMIN D IN CHRONIC HEMODIALYSIS PATIENTS
}

Jamai I, El. Boukhrissi F. and Mahmoud M

Service De Biochimie, Laboratoire Centrale d'Analyses Médicales, CHU Hassan II, Fès, Maroc.

\section{Manuscript Info}

\section{Manuscript History}

Received: 22 January 2020

Final Accepted: 25 February 2020

Published: March 2020

Key words:-

D - Deficiency - Insufficiency -Chronic

Hemodialysis-Phosphocalcic

Metabolism- Morocco

\begin{abstract}
Vitamin D metabolism disorders are common in chronic hemodialysis patients. The aim $\mathrm{f}$ our workis to determine the status of vit $\mathrm{D}$ in chronic hemodialysis patients followed at the ElGhassani dialysis center in order to bring out the correlations between this rate and the markers of phosphocalcic assessment. Our study is cross-sectional on 131 Moroccan patients, under periodic hemodialysis. We evaluated the status of vit D, as well as the correlation between the serum 25OHD level and the epidemiological and biological parameters of phosphocalcic metabolism. Our patients had an average of $25(\mathrm{OH}) \mathrm{D}$ at $22.70+/-10.48 \mathrm{ng} / \mathrm{ml} ; 90.16 \%$ of them had a Vitamin D deficiency or deficiency. There was a correlation between the serum $25(\mathrm{OH}) \mathrm{D}$ level and the female sex. The results of this study have shown that Vit D deficiency and deficiency are almost universal in the hemodialysis population, particularly in women in winter.
\end{abstract}

Copy Right, IJAR, 2020,. All rights reserved.

\section{Introduction:-}

La vitamine D (vit D) est une pro-hormone stéroïde liposoluble, à effets pléiotropiques [1]. Contrôlant environ 3\% du génome humain. [2 ]. Les troubles du métabolisme de la vit D sont classiques chez les patients en insuffisance rénale chronique (IRC). Dans les conditions normales, la vit D ; synthétisée dans la peau ou acquise par l'alimentation ; est hydroxylée en 25-hydroxyvitamineD au niveau du foie, puis convertie principalement dans le rein en 1,25-dihydroxyvitamine $\mathrm{D}$. Chez les sujets en IRC la synthèse du 1,25-dihydroxyvitamine $\mathrm{D}$ diminue en parallèle avec la diminution de la fonction rénale, à cause de la baisse de l'activité enzymatique du 1alphahydroxylase [3]. Cependant des études récentes ont démontré que cette dernière hydroxylation peut se faire dans d'autres sites : le sein, la prostate, le côlon et les macrophages [4,5]. Ainsi le taux de la 25-hydroxyvitamineD peut être normal même avec une fonction rénale altérée. Des études sur la population générale montre que entre 70\%90\%de la population souffrent d'une carence en vit D[6].Avec comme facteur de risque : l'âge, la saison, la race, la masse corporelle et le tabagisme [7,8]. Alors que peu d'étude sont mené auprès de notre population.

Le but de ce présent travail est de déterminer le statut de la Vit $\mathrm{D}$ chez les hémodialysés chroniques suivis au centre de dialyse EL GHASSANI de Fès, et de ressortir les corrélations entre le taux de la $25 \mathrm{OH}$ D et les marqueurs du bilan phosphocalcique.

Corresponding Author:- Dr. Imane Jamai Amir Address:- Service De Biochimie, Laboratoire Centrale d'Analyses Médicales, Chu Hassan II de Fès, BP 1835, Atlas, Maroc. 


\section{Patients Et Methods:- \\ Population d'étude:}

C'est une étude transversaleréalisée en Janvier 2019, incluant 131patients hémodialysés, originaires de la région de Fès et suivis dans le Centre de dialyse El Ghassani de Fès. Il s'agit d'un échantillon non randomisé.

$87 \%$ des malades sont sous supplémentation en vit D et $37 \%$ sont sous calcium, avec une mauvaise observance thérapeutique (à cause du bas niveau socio-économique).Nous avons exclus de notre étude les dossiers incomplets.

Ainsi les résultats nous informent aussi bien de la réalité métabolique que des conséquences d'éventuelles prises médicamenteuses.

\section{Paramètres biologiques étudiés:}

Le bilan biologique est réalisé par un prélèvement sanguin veineux le matin à jeun, sur tube sec ou hépariné .L'interprétation est selon les recommandations KDIGO 2017 .

La mesure de la 25 hydroxy-vitamine est effectuée par la méthode d'électro-chimiluminescence (Architect e 601, E170). Chez les patients dialysés la carence en Vit D est définie par un taux sérique de $25(\mathrm{OH}) \mathrm{D}<20 \mathrm{ng} / \mathrm{ml}$ et le déficit en Vit D par un taux sérique de 25(OH)D entre 20 et 30ng/ml .

La mesure de la PTH : est effectuée par la méthode d'électro-chimiluminescence( Architecte 601, E170) .Les valeurs normales : 134 et $603 \mathrm{pg} / \mathrm{ml}(14.7-66 \mathrm{mmol} / \mathrm{l})$.

La mesure de la calcémie : méthode colorimétrique en utilisant le colorant arsenazo -III qui réagit avec le calcium dans une solution acide pour former un complexe bleu-pourpre. La couleur qui se développe est mesurée à $660 \mathrm{~nm}$. (ARCHITECT) . Les valeurs normales : la 84 et $104 \mathrm{mg} / \mathrm{l}(2.1-2.6 \mathrm{mmol} / \mathrm{l})$.

La mesure de la phosphorémie: méthode colorimétrique. Le phosphate inorganique réagit avec le molybdate d'ammonium pour former un complexe hétéropolyacide; phosphomolybdate; l'absorbance à $340 \mathrm{~nm}$. Les valeurs normales : la phosphorémie entre 32 et $60 \mathrm{mg} / 1(0.8-1.5 \mathrm{mmol} / \mathrm{l})$.

L'albumine : le vert de bromocrésol se combine à l'albumine est forme un complexe coloré dont l'absorbance mesurée à $328 \mathrm{~nm}$ est proportionnelle à la concentration en albumine dans le spécimen. . (ARCHITECT) .Les valeurs normales :35-52 $\mathrm{g} / 1$.

Phosphatase alcaline (PAL): En milieu alcalin, les phosphatases alcalines catalysent l'hydrolyse du pnitrophénylphosphate en p-nitrophénol, suivie par la variation de l'absorbance à $405 \mathrm{~nm}$, est proportionnelle à l'activité PAL dans le spécimen. (ARCHITECT ).Les valeurs normales : 40-129U/1

\section{Analyse statistique:}

Les donnés sont saisies et traiter à l'aide du logiciel EPI info et Excel 2007. Les résultats sont exprimés sous forme de moyennes +/- écart-types.Corrélation de Pearson pour la comparaison des variables qualitatives et quantitatives. Les résultats sont considérés comme significatifs au niveau d'incertitude de $5 \%(\mathrm{p}<0,05)$.

\section{Résultats:-}

\section{Le taux de la vit D:}

Au total 131 hémodialysés chroniques sont inclus dans l'étude. Notre population est jeune, les sujets âgés ne représentent que $13,63 \%$. La moyenne de la vit $\mathrm{D}$ est de $22,70 \mathrm{ng} / \mathrm{ml}+/-10,48 \mathrm{avec}$ un minimum de 4,10 $\mathrm{ng} / \mathrm{ml}$ et maximum de $56 \mathrm{ng} / \mathrm{ml}$. Le taux de la vit $\mathrm{D}$ est statiquement plus élevé chez les femmes que chez les hommes ( 52,63 $+/-14,20 \mathrm{ng} / \mathrm{ml}$ vs $45,4+/-16,8 \mathrm{ng} / \mathrm{ml}) \quad(\mathrm{p}=0$ 008). (Tableau I)

Tableau I:- les différents paramètres en fonction du sexe.

\begin{tabular}{|ll|l|l|l|}
\hline & & Homme $(\mathbf{n = 5 8})$ & Femme $(\mathbf{n = 6 8})$ & $\mathbf{p}$ \\
\hline Age & $(\mathbf{a n s})$ & $45+/-16,8$ & $52,63+/-14,20$ & 0,008 \\
\hline PTH $\quad(\mathbf{p g} / \mathbf{m l})$ & 10006,41 & $878,48+/-612,97$ & 0,275 \\
\hline
\end{tabular}




\begin{tabular}{|l|l|l|l|}
\hline $\mathbf{2 5}(\mathbf{O H}) \mathbf{D}(\mathbf{n g} / \mathbf{m l})$ & $24,16+/-10,67$ & $21,35+/-10,18$ & 0,125 \\
\hline PAL $\quad(\mathbf{U} / \mathbf{L})$ & $182,031+/-170,90$ & $147,38+/-115,29$ & 0,172 \\
\hline Calcémie ( mg/l) & $92,23+/-9,27$ & $94,59+/-7,29$ & 0,103 \\
\hline Phosphore ( mg/dl) & $53,24+/-17,45$ & $52,69+/-17,07$ & 0,854 \\
\hline
\end{tabular}

\section{Les paramètres biochimiques en fonctions du statut de la vit $\mathrm{D}$ :}

Selon les recommandations de KDIGO 2017, 9,84\% des malades avaient un taux de vit D normal et 90,16\% ont une hypovitaminose D. $45,45 \%$ des malades ont une carence en vitD et $31,81 \%$ ont une insuffisance. Le tableau II représente les caractéristiques des patients selon le niveau de la $25 \mathrm{OH}(\mathrm{D})$.

Tableau II:- Les caractéristiques des patients en fonctions de leur statut en vit D.

\begin{tabular}{|c|c|c|c|}
\hline & Vit D normale $(\mathrm{n}=22)$ & Carence en vit $\mathrm{D}(\mathrm{n}=58)$ & $\begin{array}{l}\text { Insuffisance en vitD } \\
(\mathrm{n}=42)\end{array}$ \\
\hline (ans) & $46,38+/-16,47$ & $51,73+/-16,35$ & $46,38+/-14,42$ \\
\hline$(\mathrm{pg} / \mathrm{ml})$ & $893,91+/-561,91$ & $928,81+/-730,87$ & $991,70+/-671,71$ \\
\hline 25(OH)D $\quad(\mathrm{ng} / \mathrm{ml})$ & $39,43+/-7,17$ & $14,11+/-3,81$ & $24,36+/-2,53$ \\
\hline$(\mathrm{U} / \mathrm{L})$ & $192,33+/-154,51$ & $141,98+/-99,90$ & $187,31+/-193,89$ \\
\hline Calcémie $\quad(\mathrm{mg} / \mathrm{l})$ & $93,35+/-9,23$ & $93,07+-7,62$ & $94,36+-9,031$ \\
\hline $\begin{array}{l}\text { Phosphore } \\
(\mathrm{mg} / \mathrm{dl})\end{array}$ & $48,62+-18,05$ & $53,59+-15,97$ & $55,81+-18,68$ \\
\hline Albumine $\quad(\mathrm{g} / \mathrm{l})$ & $43,03+-5,19$ & $42,67+-4,60$ & $44,07+-4,22$ \\
\hline
\end{tabular}

En ce qui concerne le bilan phosphocalcique : 1' hyperparathyroïdie était présente chez $61,53 \%$ des patients, repartie comme suit : 66,66\% dans le groupe des patients en insuffisance et 51,66\% dans le groupe des sujets en carence. L'hyperphosphatémie est notée chez $28,46 \%$ des malades ; $23,33 \%$ dans le premier groupe et 38,09\% dans le second. L'hypocalcémie est retrouvée dans $10 \%$ cas; $5 \%$ en déficit et $4,91 \%$ en carence . La moyenne de l'albuminémie était de 43,1+- 4,71g/l avec un minimum de 33g/l et un maximum de $53 \mathrm{~g} / \mathrm{l}$. La moyenne était $42,8 \mathrm{~g} / \mathrm{l}$ chez les patients soufrant d'une carence en vit $\mathrm{D}$ et $44,04 \mathrm{~g} / \mathrm{l}$ chez les insuffisants .Dans notre étude les variations du taux de la vitD ne suivaient pas celles de l'albumine $(\mathrm{p}=0,46)$.

Les corrélations entre le taux de la vitD et les différents parameters:

En analyse univariée, aucune corrélation n'est retrouvée entre le taux de la vit D est les paramètres du bilan phosphocalcique

\section{Discussion:-}

Le taux de la vit $\mathrm{D}$ et sa corrélation avec les paramètres du métabolisme phosphocalcique est largement étudié chez la population générale, alors que peu de publications sont destinées aux hémodialysés chroniques. Cette population qui souffre déjà d’importante perturbation du métabolisme phosphocalcique. Ainsi il nous a apparu intéressant de rapporter notre état de lieu. 
Dans notre population $90 \%$ des HD souffraient d'hypovitaminose ; 45,45\% en carence et $31,81 \%$ en insuffisance. Résultat concordant avec l'étude de Mahfoud en Tunisie [9]; 41,3\% en carence et 50,8\% en insuffisance ; l'étude de Guillame en France [10] où $78 \%$ des patients sont déficitaires et au Sénégal [11] la prévalence du déficit en Vit D est de $85,9 \%$.

De nombreux travaux ont révélé la baisse de la vit D au fur et à mesure de l'aggravation de l'insuffisance rénale. Ainsi Gonzalez et al [12] ont constaté que le déficit en vit D est plus sévère chez les patients en HD que chez les IRC ne nécessitant pas un traitement de suppléance. L'étude de Dellval [13] a montré que 84\% des patients ; qui avait une carence en $25 \mathrm{OH}(\mathrm{D})$; avaient un niveau 1 d'exposition au soleil et que $85 \%$ de ceux qui avaient des niveaux adéquat, avaient une exposition niveau 3 au soleil. Soulignant ainsi l'importance de l'exposition au soleil chez les HD . Notre étude est réalisé en hiver ce qui explique la prévalence importante de l’hypovitaminose D. Bien que l'âge avancé soit un facteur de risque de la carence en vit D, nous n'avons pas pu trouver une corrélation entre l'âge et le taux de la 25OH(D)dans notre étude, même constat dans la série de Lioussfi à Rabat [14] et celle de Delvall en Argentine [13] . L'absence de corrélation pourrait avoir de nombreuses explications : faible nombre des sujets âgés dans notre série, bonne exposition au soleil de cette tranche d’âge.

Il est connu que le déficit en vit D entraine une baisse de la réabsorption intestinale du calcium( hypocalcémie), ainsi l'organisme réagit par une augmentation de la sécrétion de la PTH [14,15,16,17,18],entrainant à long terme une hyperparathyroïdie secondaire, voir même tertiaire, avec des répercussions négatives sur le squelette et la paroi des vaisseaux [14,19,20] (dépôt de calcifications vasculaires). Dans notre étude nous n’avons pas retrouvée une corrélation entre le taux de $25(\mathrm{OH}) \mathrm{D}$, PTH et la calcémie , conformément à l'étude de Delvall [13] et contrairement à l'étude de Bahn [21] . Ce résultat est attribué à la supplémentation en calcuim et en vit $\mathrm{D}$ que reçoivent nos patients.

Aussi nous ne rejoignons pas les séries de Mahfoud et de Bhan [10,21] qui identifient 1'hypoalbuminémie comme facteur de risque de l' hypovitaminose D. L'albumine est le reflet de l'état nutritionnel et donc une hypoalbuminémie traduit un faible apport alimentaire de la vitD. Etant donné que l'albumine est la principale protéine porteuse de la vitD, l’hypoalbuminémie est responsable d'une diminution de la capacité de transport de la vitD [21] ,sans oublier les pertes urinaires de l'albumine au cours du syndrome néphrotique.

\section{Conclusion:-}

La prévalence de la carence et du déficit en Vit D chez les hémodialysés Marocains est alarmante, elle est prédominante chez les femmes âgées. Des moyens de prévention et de correction devraient être entrepris chez cette population à haut risque osseux.

\section{Bibliographie:-}

1. Delanaye.P et al. Place de la vitamine D native en dialyse. NEPHRO-760; No. of Pages11.

2. Jean $\mathrm{G}$, Chazot $\mathrm{C}$. La vitamine $\mathrm{D}$ et l'insuffisance rénale chronique : les douze points essentiels. Médecine Nucléaire xxx (2015) xxx-Xxx .

3. Ishimura E et al:Serum levels of 1,25-dihydroxyvitamin D, 24,25-dihydroxyvitamin D, and 25-hydroxyvitamin $\mathrm{D}$ in nondialyzed patients with chronic renal failure. Kidney Int 55: 1019-1027, 1999.

4. Reichel H et al: Synthesis in vitro of 1,25-dihydroxyvitamin D3 and 24,25 dihydroxyvitamin D3 by interferongamma-stimulated normal human bone marrow and alveolar macrophages.J Biol Chem 262: 10931- 10937, 1987.

5. Liu PT et al: Toll-like receptor triggering of a vitamin Dmediatedhuman antimicrobial response. Science 311:1770-1773, 2006.

6. Bour A, Nejjari B : Connaissance sur la vitamine D : état des lieux de la prévalence de 1 'hypovitaminose D chez la population marocaine. Annales des sciences de la santè,N15,Vol.1 :24-31 .

7. Holick MF: High prevalence of vitamin D inadequacy and implications for health. Mayo Clin Proc 81: 353-373, 2006.

8. Hirani Vet al: Predictors of 25-hydroxyvitaminD status among adults in two British national surveys.Br $\mathrm{J}$ Nutr 101: 760-764, 2009.

9. Mahfoudh $\mathrm{H}$ et al :Déficit en calcidiol (25OH vitamine D) chez les dialysés du CHU de Sfax (sud tunisien). http://dx.doi.org/10.1016/j.nephro.2012.07.238.

10. Guillaume et al. La prescription de vitamine D chez le patient dialysé en pratique clinique. Néphrologie \& Thérapeutique (2009) 5, 520-532. 
11. Cisse. M.M et al .Prévalence de la baisse des réserves en vitamine $\mathrm{D}$ chez les sujets à peau noire en hémodialyse périodique vivant à Dakar (Sénégal) :à propos de trente-sept cas.

12. Gonza'lez EA et al: VitaminD insufficiency and deficiency in chronic kidney disease. Am J Nephrol. 2004; 24:503-510.

13. Delvalle et al:Prevalence of $25(\mathrm{OH})$ vitamin D insufficiency and deficiency in chronic kidney disease stage 5 patients on hemodialysis. Hemodialysis International 2007; 11:315-321.

14. Lioussfi $\mathrm{Z}$ et al :Évaluation du statut de la vitamine D chez les dialysés chroniques au CHU de Rabat : prévalence du déficit et corrélation avec différentes pathologies. http://dx.doi.org/10.1016/j.nephro.2012.07.220.

15. Nigwekar et al : Ergocalciferol and cholecalciferol in CKD. Am J Kidney Dis 2012;60:139-56.

16. Hewison M et al: 1alpha-Hydroxylase and the action of vitamin D. J Mol Endocrinol 2000;25:141-8.

17. Zehnder D et al: Expression of 25-hydroxyvitamin D3-1alpha-hydroxylase in the human kidney. J Am Soc Nephrol 1999;10:2465-73.

18. Levin A et al:Prevalence of abnormal serum vitamin D, PTH, calcium, and phosphorus in patients with chronic kidney disease: results of the study to evaluate early kidney disease. Kidney Int 2007;71:31-8.

19. LaClair RE et al: Prevalence of calcidiol deficiency in CKD: a cross-sectional study across latitudes in the United States. Am J Kidney Dis 2005;45:1026-33. [63]

20. KDIGO 2012 clinical practice guideline for the evaluation and management of chronic kidney disease. Kidney Int Suppl 2013;3:S1-150.

21. Bhan I et al: Clinical measures identify vit D deficiency in dialysis. Clin J Am Soc Nephrol 5: 460-467, 2010. doi: 10.2215/CJN.06440909. 